\title{
Social Cloud Computing Using Social Network
}

\author{
Harshali Sonawane ${ }^{1}$, Divya Gupta ${ }^{2}$, Ashmira Jadhav ${ }^{3}$ \\ ${ }^{\text {I Information Technology, BVCOEW/ University of Pune, India) }}$ \\ ${ }^{2}$ (Information Technology, BVCOEW/ University of Pune, India) \\ ${ }^{3}$ (Information Technology, BVCOEW/ University of Pune, India)
}

\begin{abstract}
A level of trust can be developed by online relationships in social network which are based on real world relationships. To form a dynamic Social Cloud and to enable users to share heterogeneous resources within the context of a social network we have proposed leveraging of the social relationships. We believe that combining trust relationships with suitable incentive mechanisms could provide much more sustainable resource sharing mechanisms. We have introduced a business application as a means of regulating sharing, due to the unique nature of the Social Cloud. The business application is innovative as it uses both social and economic protocols. In today's world, social community credentials are being used for authentication purpose on various other websites (e.g. Facebook). Here we outline our vision of creating a Social Storage Cloud, looking especially at possible market mechanisms that could be used to create a dynamic Cloud infrastructure in a Social network environment.
\end{abstract}

Keyword : Business Application, Cloud computing, Services computing, Social Cloud, Social networks, Web services.

\section{Introduction}

With the increasingly ubiquitous nature of Social networks and Cloud computing, users are starting to explore new ways to interact with, and exploit these developing paradigms. Social networks are used to reflect real world relationships that allow users to share information and form connections between one another, essentially creating dynamic Virtual Organizations. We propose leveraging the pre-established trust formed through friend relationships within a Social network to form a dynamic Social Cloud, enabling friends to share resources within the context of a Social network. We believe that combining trust relationships with suitable incentive mechanisms (through financial payments or bartering) could provide much more sustainable resource sharing mechanisms. This paper outlines our vision of, and experiences with, creating a Social Storage Cloud, looking especially at possible market mechanisms that could be used to create a dynamic Cloud infrastructure in a Social network environment. Social network provides us with basic means of communication. People nowadays give equal importance to real and online world relationships. In today's world social community credentials are used for authenticating on various other websites (e.g. Facebook). Social network is a platform widely used by many users. [5]

\section{Need}

Nowadays online relationships are mostly based on the social networks which are based on real world relationships. As these are real world relationships, therefore there is high level of trust between the users. So we enable users to share their resources on social networks. Social communities have extended become a everyday part of many people's lives. Social networking communities provide a platform for enhancing communication and sharing between users. Social communication sites have become more than sharing and communicating with other people, for instance a multitude applications use Facebook credentials for authenticating. [5][6]

\section{Scope}

For many people social networks provide a primary means of communication between friends, family, and coworkers. The social market is novel, as it uses both social and economic protocols to facilitate trading. We propose leveraging these relationships to form a dynamic "Social Cloud," thereby enabling users to share heterogeneous resources within the context of a social network.

In addition, the inherent socially corrective mechanisms (incentives, disincentives) can be used to enable a cloud-based framework for long term sharing with lower privacy concerns and security overheads than are present in traditional cloud environments. Due to the unique nature of the Social Cloud, a social market place is proposed as a means of regulating sharing. [5] 


\section{Social Cloud}

A Social Cloud is a scalable computing model where virtualized resources are contributed by users which are dynamically provisioned amongst a group of friends. Users may also choose to share these resources freely and make use of a reciprocal credit-based model.

\subsection{SOCIAL NETWORKING}

Users are more likely to trust information from a friend if the digital relationship between the two is based on a real world relationship (friend, family, colleagues) rather than a purely online relationship (second life, online games, etc.). Users of social networking site can add friends according to locations and university. User gets notifications via email as well as on the social networking application. When user advertises in business application, the notification is given only to the user's friends on the social networking site developing a level of trust amongst users. User can purchase the items from the notification panel. Credits will be exchanged between the seller and purchaser. Social network users can give their memory on lease to their friends on monthly basis. Notification is given to the person whose allocated memory is about to expire.

\subsection{BUSINESS APPLICATION}

The Social Marketplace is at the core of the Social Cloud and is used to regulate sharing within a group. Each group is associated with a separate instance of the market. The marketplace is tasked with allocating resources between peers according to predefined economic or noneconomic protocols. Traditionally, a marketplace is assumed to be based on the exchange of goods for money, however in a Social Cloud the marketplace is not necessarily monetary. For example, a non tangible trophy system (similar to that used in volunteer models) may provide suitable incentives to encourage sharing among friends, in this case the marketplace is responsible for managing the trophy model and regulating exchange. In general, the Social Marketplace is defined by the needs of the group and in many cases different types of market metaphors may coexist. Uploading and downloading of data can be done on the allocated memory space of the user.

\subsection{WEB SERVICES}

Web services provide the functionality to establish collaboration between social networking site and Business application. Web services retrieves contacts from social networking site which are further used in Business application.

\section{Protocols}

SOAP (Simple Object Access Protocol) is a way for a program running in one kind of operating system to communicate with a program in the same or another kind of an operating system by using the World Wide Web's Hypertext Transfer Protocol (HTTP)and its Extensible Markup Language (XML) as the mechanisms for information exchange. Since Web protocols are installed and available for use by all major operating system platforms, HTTP and XML provide an already at-hand solution to the problem of how programs running under different operating systems in a network can communicate with each other. SOAP specifies exactly how to encode an HTTP header and an XML file so that a program in one computer can call a program in another computer and pass it information. It also specifies how the called program can return a response.

In the proposed system SOAP is a protocol specification for exchanging structured information in the implementation of web services in network.

As an example of how SOAP procedures can be used, a SOAP message could be sent to a web site of social networking that has web services enabled, such as a user's and their friends on social site(friends, family and colleagues) database, with the parameters needed for a search. The site would then return an XMLformatted document with the resulting data, e.g., name, location, university. With the data being returned in a standardized machine-parse able format, it can then be integrated directly into a third-party i.e. the business application web site.

\section{System Architecture}

The system architecture describes the overall working of the modules, and their interaction with each other. The client side enables the users to interact with business application and social network. The business application provides the user, the functionalities such as memory lease, selling products. The business application uses the web services to retrieve the data from social network. The social network application will save data into social network database which will be later utilized by business application. Business application will store its information into business application database (see Fig.1). 


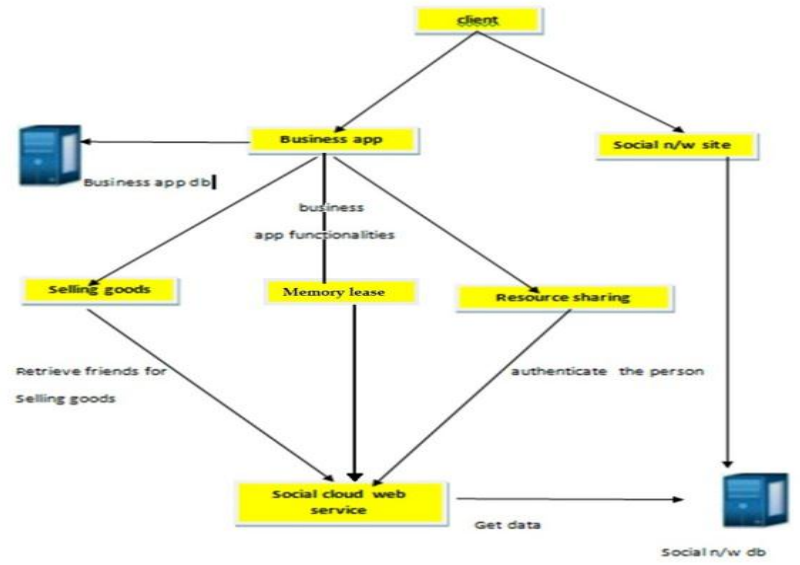

Fig.1

\section{Working Of Proposed System}

Sequence diagram shows the flow of the proposed system. It shows the working of system in a sequential manner. It interprets the system interaction from the user perspective. See Fig.2

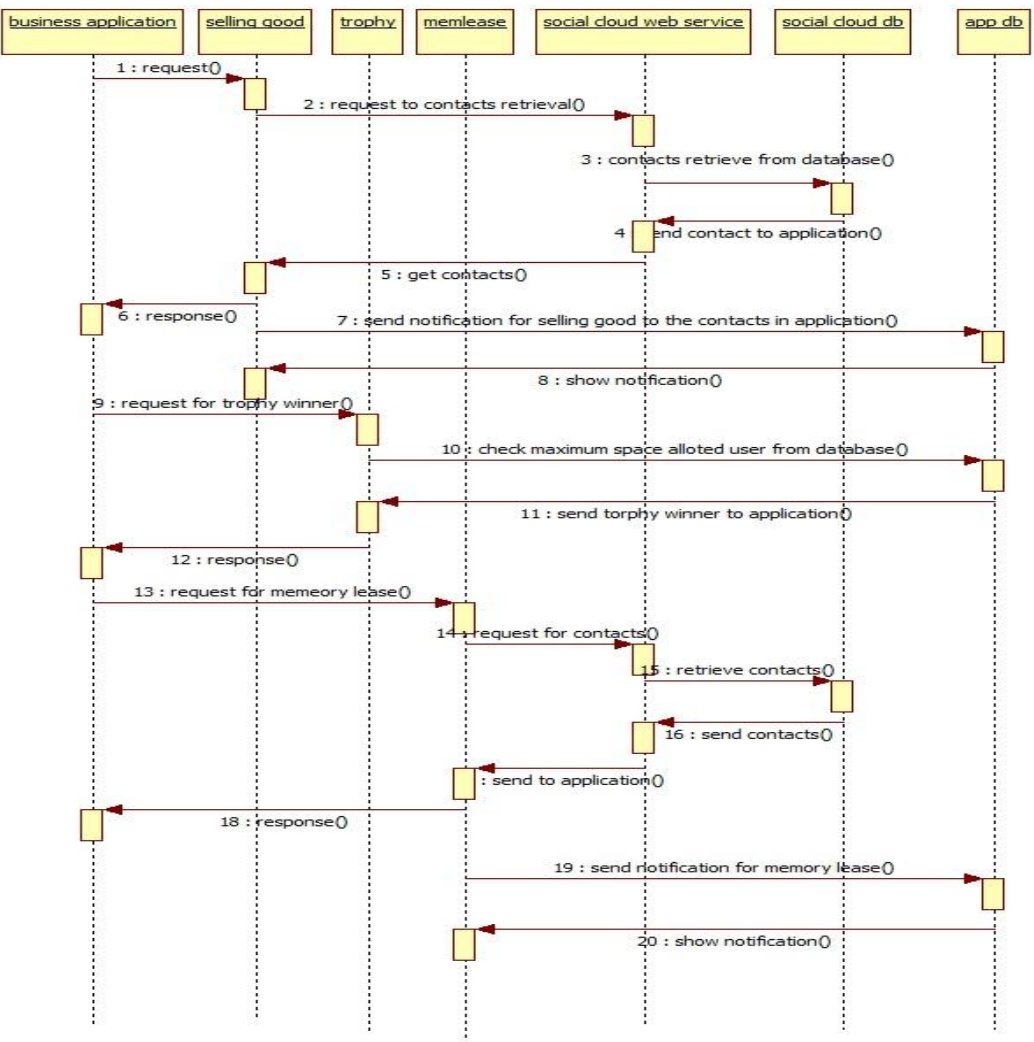

Fig.2

\section{Future Work}

Reverse auctions can be introduced to the business applications where a user1 can first upload the details of memory he/she wants to rent. Then other users can give similar memory on rent to user 1 . This may involve altering existing protocols or defining new socially oriented trading protocols. Payment gateways can be introduces instead of credit. 


\section{References}

[1] Rajkumar Buyya, Srikumar Venugopal. Grid Computing and Distributed Systems Laboratory, Department of Computer Science and Software Engineering. The University of Melbourne, Australia , IThe Gridbus Toolkit for Service Oriented Grid and Utility Computing: An Overview and Status Report"

[2] Miranda Mowbray, Francisco Brasileiroz, Nazareno Andradez, Jaindson Santanaz, Walfredo Cirne . \A Reciprocation-Based Economy for Multiple Services in Peer-to-Peer Grids" Hewlett-Packard Laboratories Bristol zUniversidade Federal de Campina Grande Filton Rd, Stoke Gi_ord Av. Aprgio Veloso, s/n - 58.109-970 Bristol BS34 8QZ, UK Campina Grande.

[3] Je_Pasternack, Waldspurger, Hogg, Huberman, Kephart, and Stornetta . ISpawn: A Distributed Computation Economy". .

[4] David P. Anderson. IBOINC: A System for Public-Resource Computing and Storage". Published in Grid Computing, 2004. Proceedings: Fifth IEEE/ACM International Workshop on 8 Nov 2004.

[5] Kyle Chard, Simon Caton, Omer Rana, and Kris Bubendorfer. ISocial Cloud: Cloud Computing in Social Networks". Published in Cloud Computing (CLOUD), 2010 IEEE 3rd International Conference on 5-10 July 2010.

[6] Kyle Chard, Member, IEEE, Kris Bubendorfer, Member, IEEE,Simon Caton, Member, IEEE, and Omer F. Rana, Member, IEEE Social Cloud Computing: \A Vision for Socially Motivated Resource Sharing". 